\title{
Extraskeletal osteosarcoma arising in the subcutaneous tissue of the lower leg: A case report and literature review
}

\author{
TAKASHI TAMURA ${ }^{1}$, KAYO SUZUKI $^{2}$, TAKETOSHI YASUDA ${ }^{2}$, SHIGEHARU NOGAMI $^{3}$, \\ KENTA WATANABE $^{2}$, MASAHIKO KANAMORI ${ }^{4}$ and TOMOATSU KIMURA ${ }^{2}$
}

${ }^{1}$ Department of Orthopaedic Surgery, Toyama Red Cross Hospital, Toyama, Toyama 930-0859; ${ }^{2}$ Department of Orthopaedic
Surgery, University of Toyama, Toyama, Toyama 930-0194; ${ }^{3}$ Department of Orthopaedic Surgery, Takaoka City Hospital,
Takaoka, Toyama 933-8550; ${ }^{4}$ Department of Human Science 1, University of Toyama, Toyama, Toyama 930-0194, Japan

Received June 7, 2018; Accepted June 26, 2018

DOI: $10.3892 / \mathrm{mco} .2018 .1662$

\begin{abstract}
Extraskeletal osteosarcoma (ESOS) is a rare soft tissue sarcoma accounting for 1-2\% of all soft tissue sarcomas. ESOS originating in the superficial (cutaneous-subcutaneous) tissue is extremely rare, and only 17 cases with subcutaneous ESOS have been reported in detail to date. The aim of the present study was to report an additional case of subcutaneous ESOS of the lower leg and review previous reports of subcutaneous ESOS, focusing on the clinical characteristics, including the MIB-1 labeling index, treatment methods and outcomes. A 79-year-old healthy man presented with a 3-year history of a painful, slowly growing mass in his right lower leg that measured $\sim 5 \mathrm{~cm}$ in greatest dimension. Excisional biopsy was performed, and ESOS was diagnosed based on the histopathological findings. A wide resection was performed when local recurrence developed. Six months after the wide resection, lung metastasis was detected. Considering the patient's age, stereotactic radiotherapy was performed without chemotherapy. The patient showed no evidence of local recurrence or new distant metastases for 2 years after the second surgery. We herein present this case of subcutaneous ESOS and review the previous 17 reported cases of subcutaneous ESOS. The 5-year survival rate of patients with subcutaneous ESOS was $78.6 \%$, which was better compared with that of ESOS cases
\end{abstract}

Correspondence to: Dr Kayo Suzuki, Department of Orthopaedic Surgery, University of Toyama, 2630 Sugitani, Toyama, Toyama 930-0194, Japan

E-mail: suzukayo@med.u-toyama.ac.jp

Abbreviations: ESOS, extraskeletal osteosarcoma; MRI, magnetic resonance imaging; CT, computed tomography; FDG-PET, F-18 2-fluoro-2-deoxy-glucose positron emission tomography; SUV, standardized uptake value; SRT, stereotactic radiotherapy; OS, overall survival; $\mathrm{CDF}$, continuously disease-free; AWD, alive with disease; RT, radiotherapy; DOC, died of other cause

Key words: extraskeletal osteosarcoma, subcutaneous, clinical characteristics, treatment, prognosis arising in deep soft tissue. Therefore, patients with subcutaneous ESOS may have a better prognosis compared with those with deep-seated ESOS, although the mean MIB-1 labeling index of subcutaneous ESOS was $24 \%$.

\section{Introduction}

Extraskeletal osteosarcoma (ESOS) is a rare soft tissue sarcoma defined as a malignant mesenchymal neoplasm composed of cells producing osteoid, bone and/or chondroid material, with no attachment to the bone or periosteum (1). ESOS accounts for 1-2\% of all soft tissue sarcomas and 2-4\% of all osteosarcomas $(1,2)$. ESOS occurs most frequently in the deep tissues of the thighs, followed by the buttocks and trunk (1). ESOS originating in the superficial (cutaneoussubcutaneous) tissue is extremely rare (3). To the best of our knowledge, only 17 cases of subcutaneous ESOS have been reported in detail to date. The prognosis of ESOS is reported to be worse compared with that of skeletal osteosarcoma (4). However, there are reports of long-term survival among the 17 cases of subcutaneous ESOS $(5,6)$. Furthermore, our patient with subcutaneous ESOS survived for 3 years with treatment. Therefore, superficial ESOS may have a better prognosis compared with deep-seated ESOS.

In this report, a new case of subcutaneous ESOS of the lower leg is presented, and previous reports of subcutaneous ESOS are reviewed, focusing on the clinical characteristics, including the MIB-1 labeling index, treatment methods and prognosis.

\section{Case report}

In April 2015, a 79-year-old healthy man presented with a painful, slowly growing mass in his lower right leg measuring $\sim 5 \mathrm{~cm}$ in greatest dimension. The mass had been present for $\sim 3$ years, and the patient had initially consulted another hospital. There was no reported history of trauma or radiation to this area. Plain radiographs revealed an irregular calcification pattern of $\sim 5 \mathrm{~cm}$ in the soft tissue of the lower right leg (Fig. 1). Magnetic resonance imaging (MRI) revealed a 5x2-cm subcutaneous mass, superficial to the fascia of the tibialis anterior muscle. The mass was hypointense on T1-weighted 
images and exhibited a heterogeneous high-intensity signal on fat-suppressed T2-weighted images (Fig. 2). Computed tomography $(\mathrm{CT})$ of the chest revealed no pulmonary lesions. Since the mass had been growing slowly, the differential diagnosis included a low-grade soft tissue sarcoma with bone mineralization, or a benign tumor. Marginal resection of the mass was performed in the previous hospital and the excised tumor was histopathologically diagnosed as ESOS; the patient was then referred to our institution for further treatment. Since the patient was elderly, it was decided not to administer chemotherapy, but to follow him up regularly.

One year after tumor resection in the previous hospital, the patient noticed a $2-\mathrm{cm}$ mass in the surgical wound. Plain radiographs revealed a mass with calcification in the soft tissue of the lower leg. MRI revealed a 1x1-cm mass in the previous surgical scar that was hypointense on T1-weighted images and exhibited a heterogeneous high-intensity signal on the fat-suppressed T2-weighted images. F-18 2-fluoro-2-deoxy-glucose positron emission tomography (FDG-PET)/CT revealed accumulation of FDG only in the palpable mass of the lower leg, with a maximum standardized uptake value of 6.7 (Fig. 3). Local recurrence of ESOS was diagnosed, and a wide resection was performed, with skin grafting from the inguinal area. On histopathological examination, the resected tumor was composed of proliferating chondrocytic cells with nuclear atypia producing coarse lacelike neoplastic bone (Fig. 4). Immunohistochemical staining for Ki-67 revealed a high proliferative index of $35 \%$. These findings led to a diagnosis of the chondroblastic type of ESOS. Since the patient was elderly, no postoperative chemotherapy was administered. A solitary lung metastasis appeared 6 months after resection of the recurrent tumor (Fig. 5A), and stereotactic radiotherapy (SRT; 60 Gy/4 fractions/5 days) was delivered to the lung metastasis. There were no complications, such as radiation pneumonitis, after SRT. Since the metastatic pulmonary lesion did not increase in size for 2 years after SRT, the lesion was considered to have undergone scarification by SRT (Fig. 5B). The patient had no evidence of local recurrence or new distant metastasis for 2 years after the second surgery at the latest follow-up date of May 14, 2018.

\section{Discussion}

ESOS mostly arises in deep soft tissues. The superficial subcutaneous tissue is rarely involved, accounting for $<10 \%$ of ESOS cases (1). A PubMed search was performed to identify cases of ESOS arising in subcutaneous tissues. The search term was 'extraskeletal osteosarcoma AND subcutaneous'. A total of 34 papers were identified, only 10 of which reported the clinical characteristics of primary subcutaneous ESOS in detail (3,5-13). A total of 17 cases were included in these 10 papers. The clinical characteristics of the 17 cases and the case presented herein are summarized in Table I. These cases were of primary ESOS arising in subcutaneous tissue, rather than occurring secondarily at the site of trauma or radiotherapy. The mean age of the 18 cases was 56.3 years (range, 15-80 years). Although ESOS is common in the fifth to seventh decades of life at diagnosis (1), $30 \%$ of cases of subcutaneous ESOS were aged $<40$ years $(6,9,11,12)$. Subcutaneous ESOS may develop in younger adults compared with deep-seated

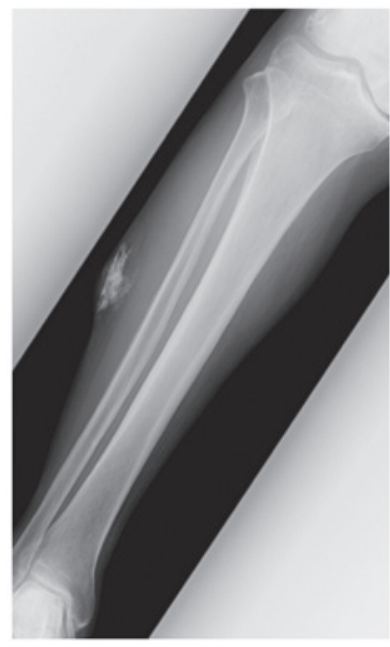

Figure 1. Anteroposterior radiograph of the lower leg before the initial operation. The radiograph shows irregular calcification within the soft tissue of the right lower leg.
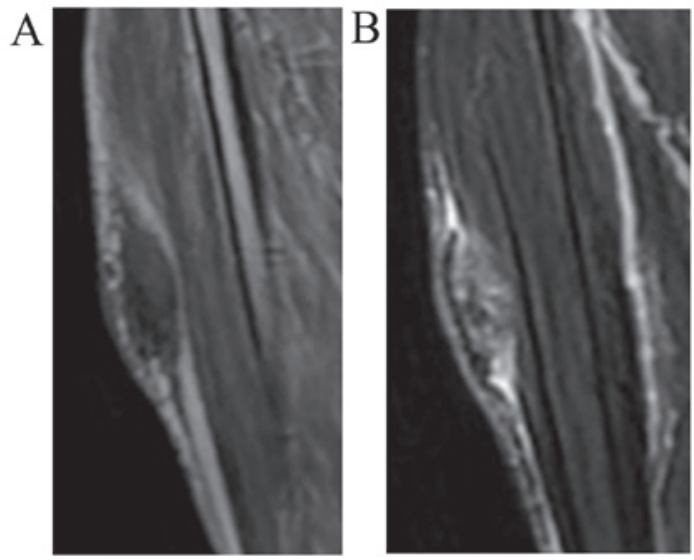

Figure 2. Magnetic resonance imaging findings at initial presentation. The subcutaneous mass was sized $5 \times 2 \mathrm{~cm}$ and located superficial to the fascia of the tibialis anterior muscle. The mass was (A) hypointense on T1-weighted images and (B) exhibited a heterogeneous high-intensity signal on fatsuppressed T2-weighted images.

ESOS. The 18 patients included 9 men and 9 women. The mean tumor size of the 18 subcutaneous ESOS cases was $4.95 \mathrm{~cm}$ (range, $1.5-15 \mathrm{~cm}$ ), and 11 tumors $(61 \%)$, including the present case, were $<5 \mathrm{~cm}$ in greatest dimension $(3,6-9,12,13)$. In the largest series of ESOS cases $(n=266), \sim 80 \%$ were tumors sized $>5 \mathrm{~cm}$ (14). Generally, it is considered that ESOS are $>5 \mathrm{~cm}$, but since subcutaneous ESOS are located more superficially, a number of patients may visit the hospital for the first time while the tumor is relatively small. The sites of tumor origin were non-extremities in 10 cases and extremities in 8 cases (6 lower and 2 upper extremities). Although the majority of ESOS cases were reported to involve the extremities (14), subcutaneous ESOS may also involve the trunk.

A preoperative diagnosis of subcutaneous ESOS was made in 9 patients; 2 cases were not diagnosed preoperatively; and there was no description in 7 cases. The preoperative diagnostic methods for 9 patients were 5 excisional biopsies, 3 needle biopsies and 1 core biopsy. With ESOS originating in 


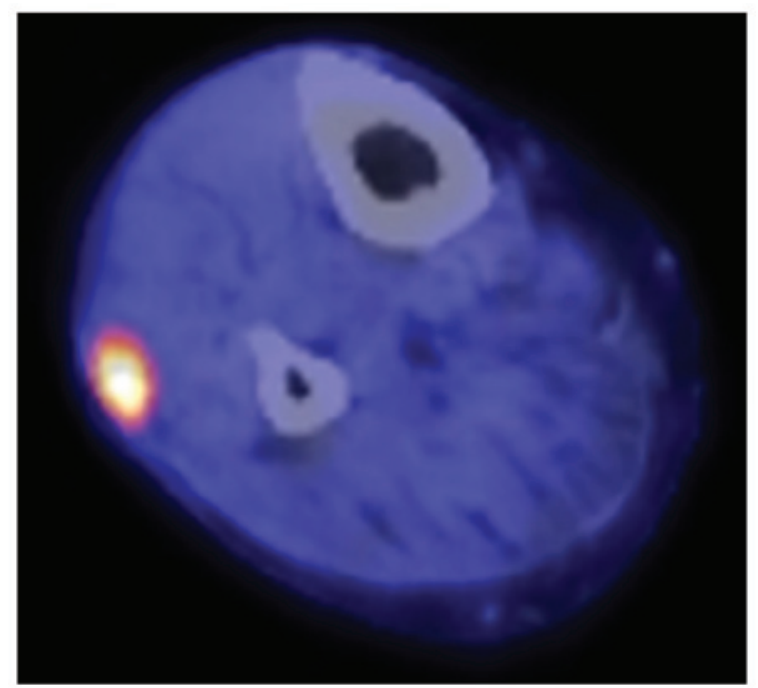

Figure 3. FDG-PET/CT images of the local recurrence after the initial resection. Increased FDG accumulation is observed in the mass at the subcutaneous tissue, with a maximum SUV of 6.7. FDG, fluorodeoxyglucose; PET, positron emission tomography; CT, computed tomography; SUV, standardized uptake value.

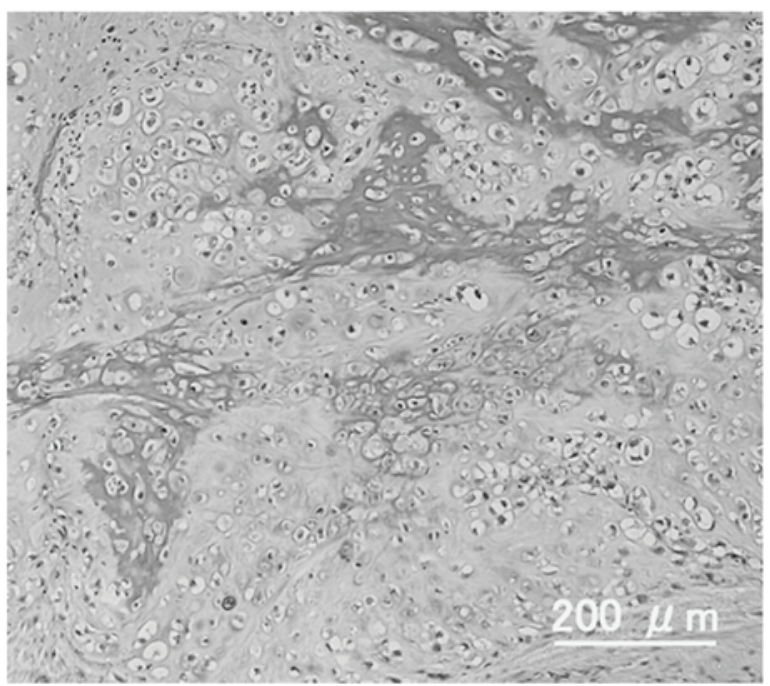

Figure 4. Histopathological findings of the resected recurrent tumor. The resected tumor was composed of proliferating chondrocytic cells with nuclear atypia and producing coarse lace-like osteoid (hematoxylin and eosin staining; magnification, $\mathrm{x} 40$ ).

the subcutaneous tissue rather than intramuscularly, the tumor is thin, and needle biopsy is difficult, even if the long axis is large. Therefore, excisional biopsy is often performed. Even in the present case, since the long diameter was $5 \mathrm{~cm}$, but the thickness was only $2 \mathrm{~cm}$, excisional biopsy was performed, and the diagnosis was obtained.

Similar to ESOS occurring in the deep soft tissue, wide resection is also the gold standard of treatment for subcutaneous ESOS (13). The effectiveness of neoadjuvant or adjuvant chemotherapy for ESOS is controversial. Treatment of subcutaneous ESOS includes surgery, chemotherapy or radiotherapy $(\mathrm{RT})$, alone or in combination $(3,6,11)$. Wide resection was performed in all but one of the 18 reported
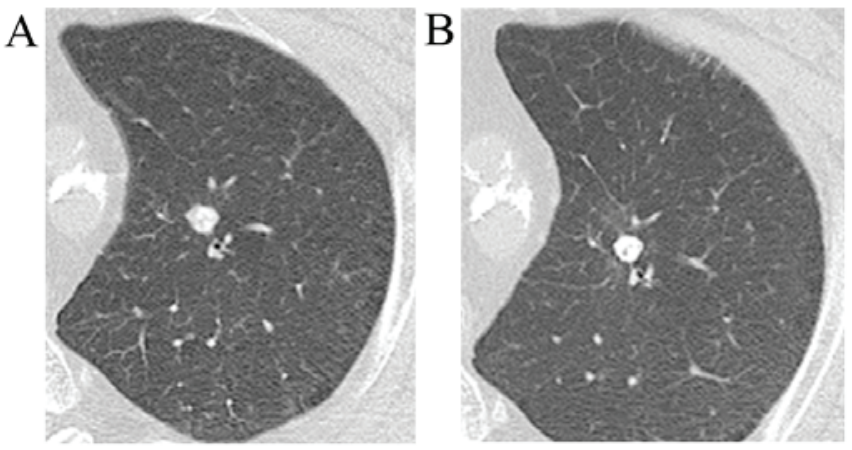

Figure 5. Chest computed tomography. (A) A single lung metastasis appeared at 6 months after resection of the recurrent leg tumor. (B) The lung metastasis exhibited no change in size within 2 years after stereotactic radiotherapy.

cases, including the present case. In the one case that did not undergo surgery, no chemotherapy was administered, and the patient only received RT (6). Chemotherapy was performed in $7(38.9 \%)$ of the 18 patients, 4 of whom received neoadjuvant chemotherapy and 3 postoperative adjuvant chemotherapy. RT as adjuvant therapy was performed in only 3 cases. A recent study of ESOS with the largest number of cases reported that the 5-year disease-free survival and overall survival (OS) were significantly better in patients treated with chemotherapy compared with those not administered chemotherapy, with a 5-year OS rate of 62 and 38\%, respectively (14). Although the number of ESOS patients was small, it was reported that the effect of chemotherapy was limited. Fan et al reported that chemotherapy with doxorubicin and ifosfamide had a positive effect on local recurrence for patients with stage III disease, but had no effect on disease-specific survival (15). The outcome of patients with subcutaneous ESOS was continuously disease-free (CDF) or alive with disease (AWD) in 14 patients [7 who received chemotherapy (6-11) and 7 who did not $(3,5-7,12,13]$; thus, the effectiveness of chemotherapy remains unclear. Further investigation of the usefulness of chemotherapy is required.

RT was performed in 4 patients (22\%). RT was administered postoperatively in 2 cases, preoperatively in 1 case and palliatively in 1 case. The role of adjuvant RT for ESOS is debatable. Choi et al reported that there was no significant difference in disease-free survival between patients treated and those not treated with radiation (16). Longhi et al also reported that RT for ESOS decreased the incidence of local relapse for patients with tumors sized $>5 \mathrm{~cm}$ and $\mathrm{R} 0$ margins, but no significant difference in local relapse rate was observed for patients with tumors $>5 \mathrm{~cm}$ and R1 margins (14). However, RT for patients with stage III $(>5 \mathrm{~cm})$ exerted a positive effect on local control, but not on disease-specific survival (15). In the present review of subcutaneous ESOS, since the number of patients who received RT was small, its effectiveness is unclear. In the present case, postoperative adjuvant chemotherapy was not performed due to the patient's age. In addition, the patient did not receive postoperative adjuvant RT, as the histopathological assessment of the resected recurrent tumor was R0.

ESOS tends to cause local recurrence and distant metastases, and localized ESOS is reported to relapse in $40-50 \%$ of 


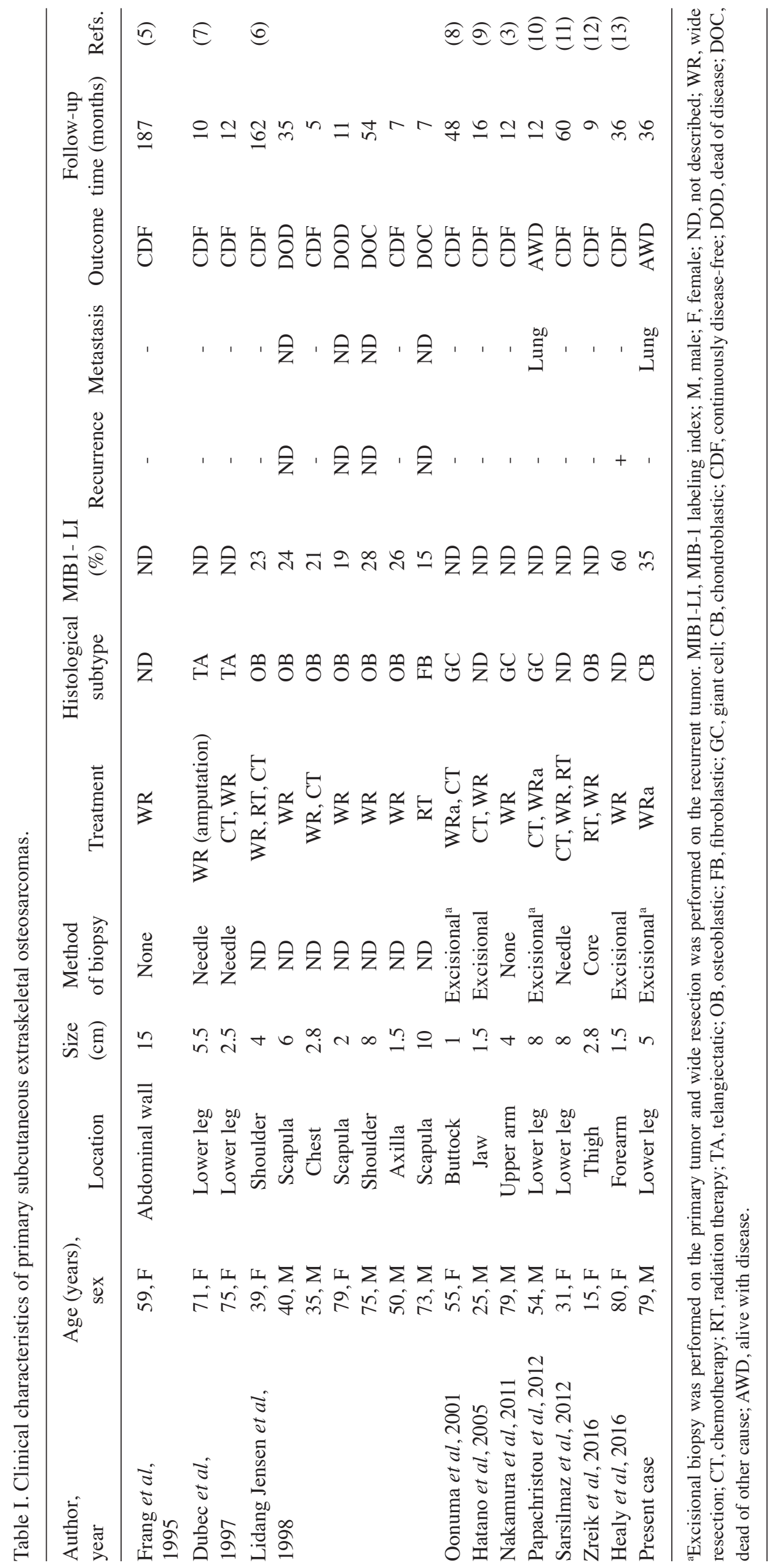


the cases $(4,14,16)$. Choi et al reported that the local recurrence rate was $19 \%$ and that of distant metastatic recurrence $38.1 \%$ among patients with localized ESOS, all of whom had lung metastasis (16). Longhi et al also reported that the local recurrence rate was $24.6 \%$, and that the distant metastatic recurrence rate was $41.7 \%$ among patients with localized ESOS (14). In the present review, only 1 case developed local recurrence 8 months after surgery (13). In these subcutaneous ESOS series, the local recurrence rate was $5.6 \%$, which was lower than the recurrence rate of previous ESOS cases. Furthermore, distant metastasis was confirmed in 2 cases $(11.1 \%)$ of subcutaneous ESOS [case reported in (10) and the present case], and its frequency was low compared with the frequency of distant metastasis in previous ESOS cases. The reports with larger numbers of cases with localized ESOS demonstrated that the 5-year survival rate was relatively poor, $\sim 50-60 \%(4,14,16)$. Thus far, only few reports have examined the prognosis of ESOS separately for ESOS cases occurring in subcutaneous tissue and those in deep tissue. In this review of patients with subcutaneous ESOS, the outcome at the final follow-up observation was relatively good: CDF in 13, AWD in 1, dead of disease in 1, and dead of other cause (DOC) in 2. The 5-year survival rate was calculated by the Kaplan-Meier method for 16 patients, excluding 2 DOC cases, among the subcutaneous ESOS cases reviewed. The 5-year survival rate of patients with subcutaneous ESOS was 78.6\%, which was better compared with that of the previous ESOS cases (Fig. 6). To the best of our knowledge, this is the first report of the survival rate only for subcutaneous ESOS cases, and the prognosis may be better for subcutaneous ESOS rather than for deep-seated ESOS. There are a few reports on the prognostic factors for ESOS. Thampi et al reported that adverse prognostic factors on multivariate analysis were axial tumor site and larger tumor size ( $\geq 10 \mathrm{~cm}$ ) (4). Choi et al also demonstrated that the prognostic factors for event-free survival of localized ESOS were significantly associated with the tumor being located superficially and having achieved R0 resection (16). The MIB-1 labeling index evaluated by immunohistochemical staining using Ki-67 was also reported to be a prognostic factor associated with survival, and it was estimated that a value of $<24 \%$ would reflect a good prognosis (6). The mean MIB-1 labeling index with subcutaneous ESOS was 24\% (range, 15-60\%, n=9). The MIB-1 labeling index of the present case was 35\%, which was higher than the mean in the subcutaneous ESOS series. This value of the MIB-1 labeling index corresponds to a poor prognostic factor for previous ESOS cases, but our patient has survived for 3 years as CDF.

Subcutaneous ESOS is considered to be highly malignant histopathologically (1), but it is rare for a superficial tumor to invade into the deep tissue, and it is often found at a size of $\leq 10 \mathrm{~cm}$, so that wide resection can be performed; thus, the prognosis of subcutaneous ESOS is considered to be better compared with that of ESOS arising from deeper tissues. Therefore, further information on the necessity of chemotherapy and the prognosis of subcutaneous ESOS is required.

In summary, a new case of ESOS arising in the subcutaneous tissue of the lower leg was reported. The diagnosis of subcutaneous ESOS was made by excisional biopsy, and wide resection was performed at the time of tumor recurrence. The tumor was $5 \mathrm{~cm}$ in greatest dimension, and the MIB-1 labeling index was $35 \%$. Although the patient developed a solitary lung

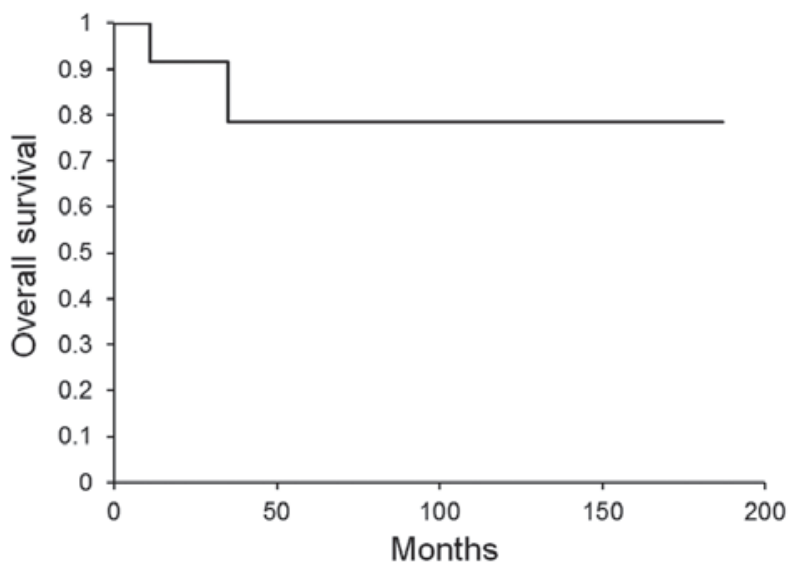

Figure 6. Overall survival rate of the reviewed cases of subcutaneous ESOS The 5-year survival rate of 18 patients with subcutaneous ESOS was estimated at $78.6 \%$ by the Kaplan-Meier method. ESOS, extraskeletal osteosarcoma.

metastasis, the lesion was successfully controlled by SRT without chemotherapy, and the patient survived for 3 years as CDF. Furthermore, the previous subcutaneous ESOS cases were reviewed herein, focusing on the clinical characteristics, MIB-1 labeling index and outcomes. Patients with subcutaneous ESOS may have a better prognosis compared with those with ESOS arising from deeper soft tissue, although the mean MIB-1 labeling index of subcutaneous ESOS was 24\%.

\section{Acknowledgements}

The authors are grateful to Professor Joji Imura, Department of Pathology, University of Toyama (Toyama, Japan), for discussion of histopathological diagnosis.

\section{Funding}

The present study was supported by the Japan Society for the Promotion of Science (JSPS; grant no. JP17K16681).

\section{Availability of data and materials}

All data generated or analyzed during this study are included in this published article.

\section{Authors' contributions}

TT and KS made substantial contributions to the conception and design. TY was responsible for the acquisition or analysis and interpretation of data. KW provided advice on the data analysis. KS, TY and SN were involved in surgical treatment. MK and TK were involved in drafting the manuscript or revising it critically for important intellectual content. KS made a critical revision of the article for important intellectual content. All authors have read and approved the final version of the manuscript.

\section{Ethics approval and consent to participate}

This report was approved by the Ethics Committee of the University of Toyama (Toyama, Japan), and clinical research number '21-22' was granted. 


\section{Patient consent for publication}

Written informed consent was obtained from the patient for publication of this report and accompanying images. A copy of the written consent is available for review upon request.

\section{Competing interests}

The authors declare that they have no competing interests.

\section{References}

1. Rosenberg AE: Extraskeletal osteosarcoma. In: WHO classification of tumours of soft tissue and bone. Fletcher CDM, Bridge JA, Hogendoorn PCW and Mertens F (eds). IARC, Lyon, pp161-162, 2013

2. Chung EB and Enzinger FM: Extraskeletal osteosarcoma. Cancer 60: 1132-1142, 1987.

3. Nakamura T, Matsumine A, Nishimura K, Yokoyama H, Murata T, Uchida A and Sudo A: Extraskeletal subcutaneous osteosarcoma of the upper arm: A case report. Oncol Lett 2: 75-77, 2011.

4. Thampi S, Matthay KK, Boscardin WJ, Goldsby R and DuBois SG: Clinical features and outcomes differ between skeletal and extraskeletal osteosarcoma. Sarcoma 2014: 902620, 2014.

5. Fang Z, Yokoyama R, Mukai K, Beppu Y and Fukuma H: Extraskeletal osteosarcoma: A clinicopathologic study of four cases. Jpn J Clin Oncol 25: 55-60, 1995.

6. Lidang Jensen M, Schumacher B, Myhre Jensen O, Steen Nielsen $O$ and Keller J: Extraskeletal osteosarcomas: A clinicopathologic study of 25 cases. Am J Surg Pathol 22: 588-594, 1998.

7. Dubec JJ, Munk PL, O'Connell JX, Lee MJ, Janzen D, Connell D, Masri B and Logan PM: Soft tissue osteosarcoma with telangiectatic features: MR imaging findings in two cases. Skeletal Radiol 26: 732-736, 1997.
8. Oonuma M, Hatori M, Hosaka M and Kokubun S: Extraskeletal osteosarcoma arising in the buttock. Ups J Med Sci 106: 211-215, 2001.

9. Hatano H, Morita T, Kobayashi H, Ito T, Segawa $\mathrm{H}$ and Hasegawa S: Extraskeletal osteosarcoma of the jaw. Skeletal Radiol 34: 171-175, 2005.

10. Papachristou DJ, Goodman M, Cieply K and Rao UN: Extraskeletal osteosarcoma of subcutaneous soft tissue with lymph node and skin metastasis: A case report with fluorescence in situ hybridization analysis. Pathol Oncol Res 18: 107-110,2012.

11. Sarsilmaz A, Argin M, Sezak M, Altay C and Erdogan N: Primary osteosarcoma arising from subcutaneous tissue: 5-year follow-up. Clin Imaging 36: 402-405, 2012.

12. Zreik RT, Meyer RG, Jenkins RB, Norambuena GA and Fritchie KJ: A rare pediatric example of subcutaneous extraskeletal osteosarcoma: A case report and review of the morphologic differential diagnosis. Am J Dermatopathol 38: e44-e48, 2016.

13. Healy C, Kahn LB and Kenan S: Subcutaneous extraskeletal osteosarcoma of the forearm: A case report and review of the literature. Skeletal Radiol 45: 1307-1311, 2016.

14. Longhi A, Bielack SS, Grimer R, Whelan J, Windhager R, Leithner A, Gronchi A, Biau D, Jutte P, Krieg AH, et al: Extraskeletal osteosarcoma: A European Musculoskeletal Oncology Society study on 266 patients. Eur J Cancer 74: 9-16, 2017.

15. Fan Z, Patel S, Lewis VO, Guadagnolo BA and Lin PP: Should high-grade extraosseous osteosarcoma be treated with multimodality therapy like other soft tissue sarcomas? Clin Orthop Relat Res 473: 3604-3611, 2015.

16. Choi LE, Healey JH, Kuk D and Brennan MF: Analysis of outcomes in extraskeletal osteosarcoma: A review of fifty-three cases. J Bone Joint Surg Am 96: e2, 1-8, 2014. 